\title{
Propriedades físico-hídricas do solo sob plantio direto escarificado e rendimento da soja ${ }^{1}$
}

\author{
Soil physico-hidrical properties under chiseled no-tillage and soybean yield
}

\author{
Rodrigo Kurylo Camara ${ }^{2}$ Vilson Antonio Klein ${ }^{3}$
}

\section{RESUMO}

\begin{abstract}
O solo sob plantio direto $(P D)$ sofre alterações ocasionados pela mínima mobilização e pelo tráfego de máquinas. $O$ efeito dessas alterações sobre o desenvolvimento das plantas é dependente das condições climáticas. A escarificação esporádica do solo sob PD tem se apresentado como uma alternativa para minimizar os efeitos da compactação, especificamente em períodos de deficiência hídrica. $O$ objetivo deste trabalho foi avaliar as alterações nas propriedades físicohídricas de solo sob PD e plantio direto escarificado (PDE) bem como o desempenho da cultura da soja implantada em semeadura direta com dois mecanismos sulcadores na semeadora. $O$ experimento foi instalado em área com seis anos sob PD, sendo que parte da área foi escarificada. Avaliou-se o teor de matéria orgânica, a densidade do solo, a máxima densidade e a densidade relativa, a resistência mecânica do solo à penetração, os componentes de rendimento e rendimento de grãos da cultura da soja. Os resultados indicaram que a escarificação do solo sob $P D$ não afeta o teor de matéria orgânica do solo, diminui a densidade do solo, a densidade máxima, a densidade relativa e a resistência mecânica do solo à penetração. Os componentes de rendimento de grãos da cultura da soja não foram afetados pelos manejos de solo, tampouco pelos mecanismos sulcadores da semeadora. $O$ rendimento de grãos da soja também não foi afetado pelos manejos, mas positivamente pela utilização do sulcador tipo guilhotina em PD.
\end{abstract}

Palavras-chave: compactação do solo, resistência à penetração, manejo do solo.

\section{ABSTRACT}

Under no-tillage (NT) system, the soil is susceptible to changes in its structure caused by minimum soil disturbance and machine traffic. The effect of these changes on plant growth depends on climatic conditions. Sporadic soil chiseling under the NT system has been used as an option to minimize the effects of soil compaction, particularly in periods of water shortage. The aim of the present study is to analyze the changes in the soil physical and hydraulic properties under the NT system and the chiseled no-tillage (CNT) system, as well as the effect of two types of no-till drills on soybean yield. This experiment was carried out in an area in which NT had been used for six years and part of which had been chiseled. Organic matter content, bulk and particle density, relative density, soil mechanical resistance to penetration, soybean yield components, and soybean grain yield were evaluated. The results indicated that soil chiseling under NT does not affect soil organic matter content and decreases bulk and particle density, relative density, and soil mechanical resistance to penetration. Soybean grain yield components were not affected by soil management or by the use of seed drills. Soybean grain yield was not affected by soil management, but was positively affected by the use of hoetype drills under NT.

Key words: soil compaction, resistance to penetration, soil management.

\section{INTRODUÇÃO}

A exploração agrícola necessita se tornar cada vez mais sustentável. Nesse contexto, o sistema plantio direto se apresenta como um sistema conservacionista que apresenta inúmeras vantagens. No entanto, o solo sob PD apresenta maior densidade ocasionada pela sua reduzida movimentação, restrita à linha de semeadura e freqüência de tráfego, sendo esse um dos principais problemas do sistema, tendo como conseqüência a queda no rendimento das

${ }^{1}$ Parte da Dissertação do primeiro autor, financiado pela Fapergs (processo n. 01/1706.0).

${ }^{2}$ Pós-graduação em Agronomia, área de concentração Produção Vegetal, passo Fundo, RS, Brasil.

${ }^{3}$ Faculdade de Agronomia e Medicina Veterinária da Universidade de Passo Fundo (UPF), 99001-970 Passo Fundo, RS, Brasil. Email: vaklein@upf.br. Aautor para correspondência. 
culturas devido principalmente ao impedimento mecânico ao crescimento das raízes (TORMENA \& ROLOFF, 1996; GOEDERT et al., 2002). Tal fato tem motivado alguns agricultores, ainda que temporariamente, a retornar ao preparo convencional, visando corrigir essa limitação.

A compactação do solo é considerada por FREITAS (1994) a maior limitação à alta produtividade das culturas em todo o mundo, pois afeta diretamente o crescimento das raízes, diminui a capacidade de infiltração de água no solo, reduz a translocação de nutrientes, resultando em uma pequena camada para ser explorada pelas raízes. Destaca ainda que, por depender de vários fatores, principalmente a umidade do solo no período de crescimento das raízes, o efeito da compactação na produção das culturas é difícil de ser quantificado.

A porosidade de aeração e a resistência mecânica à penetração são as propriedades que mais variam com as alterações na densidade do solo (KLEIN \& LIBARDI, 2000), enquanto CAMARGO \& ALLEONI (1997) destacam que, com a diminuição da porosidade de aeração, ocorre uma redução na difusão de oxigênio.

Um parâmetro que pode ser utilizado como um indicador de compactação é a densidade relativa (DR), obtida através da relação entre a densidade do solo e a densidade máxima do solo obtida em laboratório (KLEIN, 2002). Para um Latossolo Roxo, de textura argilosa, o valor de DR em que as condições ao desenvolvimento das plantas são ótimas é de 0,715. Em solos da Escandinávia e no oeste do Canadá, a DR ótima foi de 0,77 e 0,84, respectivamente (KAY, 2000). CARTER (1990) afirma que, quando a DR supera 0,86 a 0,90 (dependendo da textura do solo), ocorre uma grande redução nos macroporos, afetando o movimento dos fluídos, e estes estariam relacionados com um menor crescimento e rendimento das culturas. FERRERAS et al. (2001) observaram, em PD, uma DR de 0,87 e, no plantio direto escarificado (PDE), de 0,85, refletindo drasticamente no rendimento da cultura de soja.

A operação de descompactação tem por objetivo aumentar a porosidade, reduzir a densidade e elevar a estabilidade de seus agregados, ao mesmo tempo em que rompe as camadas superficiais encrostadas e camadas subsuperficiais compactadas. Em função disso, a descompactação facilita o desenvolvimento radicular das plantas, eleva a taxa de infiltração e a capacidade de armazenamento de água, aumenta a permeabilidade do solo (KOCHHANN \& DENARDIN, 2000) e reduz a resistência mecânica do solo à penetração das raízes (INOUE et al., 2002).
O efeito da escarificação sob um Latossolo Vermelho, de textura argilosa foi estudado por RALISCH et al. (2001), que concluíram que essa operação resulta em efeitos imediatos na redução da resistência do solo à penetração. Comparando a utilização de escarificadores em relação ao PD, SECCO \& REINERT (1997) concluíram que o uso desses proporcionou uma melhor porosidade total do solo e maior rugosidade superficial, e estas condições têm um efeito residual pelo menos até 10 meses após o preparo. PIERCE et al. (1992) constataram o efeito residual da escarificação em um solo franco-arenoso após dois anos, concluindo que esse efeito pode diminuir, mas persiste.

Em outro estudo, SECCO et al. (1996) não observaram diferença de rendimento de grãos da soja, quando compararam vários sistemas de manejo de solo entre eles o PD e o PDE. Em outro trabalho comparando o uso de diferentes escarificadores em relação ao PD, SECCO \& REINERT (1997) observaram aumento de rendimento em milho no solo escarificado.

O rendimento de soja sob PD foi $47,88 \%$ inferior ao PDE provavelmente devido à compactação no sistema PD, reduzindo o desenvolvimento radicular em função do aumento da resistência mecânica que afeta a absorção de água e nutrientes (FERRERAS et al.,2001), enquanto CAMARGO \& ALLEONI (1997) destacam que, em solos compactados, a baixa aeração induz a ramificação das raízes adventícias superficiais, tornando-as menos eficientes na absorção de água e nutrientes.

O objetivo deste trabalho foi avaliar as propriedades físico-hídricas de solo sob PD e PDE e o desempenho da cultura da soja implantada em semeadura direta com dois mecanismos sulcadores na semeadora.

\section{MATERIAL E MÉTODOS}

O experimento foi instalado em um Latossolo Vermelho Distrófico típico, relevo ondulado e substrato basalto, com $0,61 \mathrm{~kg} \mathrm{~kg}^{-1}$ de argila, $0,10 \mathrm{~kg} \mathrm{~kg}^{-1}$ de silte e $0,29 \mathrm{~kg} \mathrm{~kg}^{-1}$ de areia. A área vinha sendo conduzida sob sistema PD contínuo por um período de 6 anos, apresentando a seguinte seqüência de culturas: 1997/98 soja-trigo, 1998/99 soja-aveia, 1999/ 00 milho-aveia, 2000/01 soja-aveia, 2001/02 milho silagem e milho. Após a colheita do milho, realizou-se a escarificação em parte da área utilizando escarificador equipado com cinco hastes parabólicas, discos de corte e com rolo destorroador/nivelador, a uma profundidade média de $25 \mathrm{~cm}$. Após a escarificação sem 
preparo complementar, semeou-se aveia preta em toda a área com a finalidade de adubação verde, utilizando semeadora-adubadora, equipada com duplo-discos (DD).

O delineamento experimental foi em faixas com parcelas subdivididas e 7 repetições, tendo como parcelas principais o PD e PDE, e as subparcelas constituídas pelas profundidades de coleta, as amostras e os mecanismos sulcadores da semeadora. A semeadora foi equipada com dois sulcadores distintos para deposição de adubo: o sulcador do tipo guilhotina $(\mathrm{G})$ que é composto de um disco de corte e um cinzel sulcador e o outro um sulcador duplo disco (DD) composto de dois discos com diâmetros diferentes desencontrados, tendo função simultânea de corte e abertura de sulco.

As amostras para determinação da densidade do solo, retenção de água, resistência mecânica do solo à penetração foram coletadas nas profundidades de 2,$5 ; 5,0 ; 10,0 ; 15,0 ; 20,0$ e $25,0 \mathrm{~cm}$, utilizando amostrador do tipo "Uhland", com cilindros com $5 \mathrm{~cm}$ de diâmetro e $5 \mathrm{~cm}$ de altura.

Determinou-se o teor de carbono orgânico do solo através do método de Walkley - Black, baseado no princípio da oxidação da matéria orgânica, conforme a metodologia descrita pela TEDESCO et al. (1995). A densidade do solo foi determinada conforme metodologia descrita pela EMBRAPA (1997) e a densidade máxima através do teste de Proctor normal com $560 \mathrm{kPa}$ (NOGUEIRA, 1998).

A resistência mecânica do solo à penetração (RP) foi determinada em laboratório, utilizando um penetrômetro eletrônico. As determinações foram realizadas em amostras com estrutura preservadas submetidas a diferentes tensões $(0 ; 6 ; 10 ; 14,5 ; 25$; $50 ; 100 ; 200 \mathrm{kPa})$. Para cada amostra, foram utilizados cerca de 200 valores centrais sendo os valores de RP ajustados a um modelo não-linear proposta por BUSSCHER (1990), que ajusta a RP em relação à densidade e umidade do solo.

A semeadura da soja foi efetuada no dia 14/ 12/2002 com uma semeadora-adubadora, com espaçamento nas entrelinhas de $0,42 \mathrm{~m}$ operando a velocidade de $3,5 \mathrm{~km} \mathrm{~h}^{-1}$. A estatura das plantas foi determinada quando a cultura se encontrava no estádio de floração plena e a colheita foi realizada com uma colhedora de parcelas, momento no qual coletaramse plantas para a determinação dos componentes de rendimento.

\section{RESULTADOS E DISCUSSÃO}

O teor de matéria orgânica (MO) no solo (Tabela 1) apresentou diferença significativa entre os dois manejos, tendo o PDE apresentado valores maiores do que o PD. Em profundidade, o PDE apresentou uma variação menor entre as camadas do que $\mathrm{PD}$, o que indica que houve uma certa redistribuição dos restos culturais da superfície em profundidade e um possível maior desenvolvimento do sistema radicular da aveia preta em profundidade.

No PD, a profundidade de $2,5 \mathrm{~cm}$ foi a que apresentou maior quantidade de $\mathrm{MO}$, enquanto, no PDE, foi nas camadas de 2,5 a 20,0cm. Comparando os sistemas nas profundidades, o PD foi igual estatisticamente ao PDE nas profundidades de 2,5 e $5,0 \mathrm{~cm}$, enquanto, nas demais profundidades, o PDE apresentou teores de MO superior ao PD.

Esses resultados indicam, ao contrário do que afirmam muitos autores, (BAYER \&

Tabela 1 - Teor de matéria orgânica a base de volume, densidade do solo e densidade relativa em função do manejo e da profundidade do solo

\begin{tabular}{|c|c|c|c|c|c|c|}
\hline \multirow{2}{*}{$\begin{array}{l}\text { Profundidade } \\
\mathrm{cm}\end{array}$} & \multicolumn{2}{|l|}{$\mathrm{PD}^{1}$} & \multicolumn{2}{|c|}{$\mathrm{PDE}^{2}$} & \multicolumn{2}{|c|}{ Média } \\
\hline & \multicolumn{6}{|c|}{ Matéria orgânica \% } \\
\hline 2,5 & A 4,12 & $\mathrm{a}$ & A 4,05 & $\mathrm{a}$ & & \\
\hline 5,0 & A 3,72 & $\mathrm{~b}$ & A 4,06 & a & & \\
\hline 10,0 & B 3,41 & $\mathrm{bc}$ & A 3,98 & $a b$ & & \\
\hline 15,0 & B 3,09 & $\mathrm{~cd}$ & A 4,02 & $a b$ & & \\
\hline 20,0 & B 2,93 & $\mathrm{~d}$ & A 3,70 & $a b$ & & \\
\hline 25,0 & B 2,87 & $\mathrm{~d}$ & A 3,54 & $\mathrm{~b}$ & & \\
\hline Média & 3,36 & & 3,89 & & & \\
\hline $\mathrm{CV}$ & & & $9,02 \%$ & & & \\
\hline $\mathrm{cm}$ & \multicolumn{6}{|c|}{ Densidade do solo $\left(\mathrm{Mg} \mathrm{m}^{-3}\right)$} \\
\hline 2,5 & 1,284 & & \multicolumn{2}{|c|}{1,171} & 1,227 & $\mathrm{~b}$ \\
\hline 5,0 & 1,340 & & \multicolumn{2}{|c|}{1,241} & 1,291 & c \\
\hline 10,0 & 1,346 & & \multicolumn{2}{|c|}{1,293} & 1,320 & c \\
\hline 15,0 & 1,339 & & \multicolumn{2}{|c|}{1,316} & 1,328 & c \\
\hline 20,0 & 1,314 & & \multicolumn{2}{|c|}{1,298} & 1,306 & c \\
\hline 25,0 & 1,277 & & \multicolumn{2}{|c|}{1,287} & 1,282 & a \\
\hline Média & A 1,317 & & \multicolumn{4}{|l|}{ B 1,268} \\
\hline $\mathrm{CV}$ & \multicolumn{6}{|c|}{$5,19 \%$} \\
\hline $\mathrm{cm}$ & \multicolumn{6}{|c|}{ Densidade relativa } \\
\hline 2,5 & A 0,845 & $\mathrm{a}$ & B 0,776 & $\mathrm{~b}$ & & \\
\hline 5,0 & A 0,865 & $\mathrm{a}$ & A 0,811 & $\mathrm{~b}$ & & \\
\hline 10,0 & A 0,863 & $\mathrm{a}$ & A 0,851 & $\mathrm{a}$ & & \\
\hline 15,0 & A 0,870 & $\mathrm{a}$ & A 0,866 & $\mathrm{a}$ & & \\
\hline 20,0 & A 0,859 & $\mathrm{a}$ & A 0,871 & $\mathrm{a}$ & & \\
\hline 25,0 & A 0,840 & $\mathrm{a}$ & A 0,858 & $\mathrm{a}$ & & \\
\hline Média & 0,857 & & 0,839 & & & \\
\hline $\mathrm{CV}$ & & & $5,18 \%$ & & & \\
\hline
\end{tabular}

${ }^{1}$ Plantio direto

${ }^{2}$ Plantio direto escarificado

Médias antecedidas pela mesma letra maiúscula na horizontal e seguidas pela mesma letra minúscula na vertical não diferem pelo teste de Duncan a 5\% de probabilidade de erro. 
MIELNICZUCK, 1997; CARVALHO et al., 1999 e FERRERAS et al., 2001), que o preparo do solo reduz o teor de MO no solo, indicando que a escarificação, pelo pouco revolvimento do solo e conseqüente pouca incorporação dos restos culturais, pode melhorar a estrutura do solo aumentando o teor de MO.

Observou-se que os valores de densidade do solo na média dos manejos (Tabela 1) apresentaram diferença significativa entre os manejos. O PDE apresentou uma densidade do solo média 3,72\% inferior ao $\mathrm{PD}$, mesmo após uma cultura de inverno (6 meses), contradizendo RALISCH et al. (2001), que concluíram que a escarificação tem efeito somente para uma cultura, não tendo efeito residual para outras culturas subseqüente, enquanto PIERCE et al. (1992) relata que o efeito da escarificação pode persistir por anos em um solo franco-arenoso.

Quanto à densidade do solo em profundidade, não foi verificada diferença significativa entre os manejos. Nota-se que a escarificação provoca uma redução do valor médio da densidade do solo e, independente do manejo do solo, a densidade foi menor na camada superficial de $2,5 \mathrm{~cm}$. Não houve interação entre sistemas de manejo e profundidades.

A densidade do solo no PD foi mais elevada que no PDE também em trabalho realizado por MACHADO et al. (1996). Isso ocorre porque o escarificador promove a perturbação do solo, ocasionando o empolamento com aumento da porosidade total (KLEIN \& BOLLER, 1995).

A densidade máxima do solo (DMS) foi semelhante entre as profundidades e os manejos $\left(1,54 \mathrm{Mg} \mathrm{m}^{-3}\right.$ para o PD e $1,51 \mathrm{Mg} \mathrm{m}^{-3}$ para o PDE), e a umidade ótima de compactação foi igual $0,25 \mathrm{~kg} \mathrm{~kg}^{-1}$. A DMS foi menor no PDE, supostamente pelo efeito do maior teor de MO, pois, conforme BRAIDA et al. (2003), o acúmulo de $\mathrm{MO}$ no solo reduz a densidade máxima do solo e aumenta a umidade crítica da máxima compactação, ressaltando que a magnitude é dependente da granulometria do solo, sendo maior em solos arenosos.

Estudando o processo de compactação DIAS JUNIOR \& MIRANDA (2000) demonstraram que as classes de solos apresentam comportamento distinto devido à variação de textura, matéria orgânica e umidade, encontrando em um Latossolo Vermelhoamarelo com teor de argila de $0,37 \mathrm{~kg} \mathrm{~kg}^{-1}$, uma DMS de $1,57 \mathrm{Mg} \mathrm{m}^{-3}$, e uma umidade ótima de $0,20 \mathrm{~kg} \mathrm{~kg}^{-1}$, e num Latossolo Vermelho-escuro com $0,57 \mathrm{~kg} \mathrm{~kg}^{-1}$ de argila, uma DMS de $1,44 \mathrm{Mg} \mathrm{m}^{-3}$, e uma umidade ótima de compactação de $0,26 \mathrm{~kg} \mathrm{~kg}^{-1}$.

A DR no campo foi maior no PD do que no PDE (Tabela 1) apresentando diferença entre manejos e profundidades. Comparando os manejos nas profundidades, o PD apresentou uma DR superior ao $\mathrm{PDE}$ apenas na profundidade de $2,5 \mathrm{~cm}$, indicando um certo nível de compactação do solo nesta profundidade. No PDE, a DR foi menor nas profundidades de 2,5 e $5,0 \mathrm{~cm}$, sendo que, nas demais profundidades, não diferiram. No entanto, no $\mathrm{PD}$, a DR não diferiu entre as profundidades, indicando uma maior uniformidade da densidade do solo em profundidade.

Em um Latossolo Roxo, de textura argilosa, o valor de DR em que as condições ao desenvolvimento das plantas seriam ótimas foi de 0,715 (KLEIN, 2002). FERRERAS et al. (2001) observaram, em um solo siltoso sob PD uma DR de 0,82 e 0,85 nas camadas de $0-6 \mathrm{~cm}$ e $10-16 \mathrm{~cm}$, respectivamente, e no PD escarificado na ordem de 0,69 e 0,85 , nas mesmas camadas, refletindo drasticamente no rendimento da cultura da soja com a pequena diferença encontrada na camada de $10-16 \mathrm{~cm}$.

A RP na capacidade de campo e no ponto de murcha permanente apresentou diferença entre os manejos do solo (Tabela 2). O PD apresentou uma RP

Tabela 2 - Resistência mecânica do solo à penetração na umidade correspondente $\left(\mathrm{m}^{3} \mathrm{~m}^{-3}\right)$ à capacidade de campo $(\mathrm{CC}=6 \mathrm{kPa})$ e ao ponto de murcha permanente $(\mathrm{PMP}=1500 \mathrm{kPa})$ em função do manejo e da profundidade do solo

\begin{tabular}{|c|c|c|c|c|c|}
\hline \multirow{2}{*}{$\begin{array}{l}\text { Profundidade } \\
\mathrm{cm}\end{array}$} & \multicolumn{2}{|l|}{$\mathrm{PD}^{1}$} & \multicolumn{2}{|c|}{$\mathrm{PDE}^{2}$} & \multirow[t]{2}{*}{ Médias } \\
\hline & \multicolumn{4}{|c|}{ Capacidade de campo } & \\
\hline 2,5 & A 1,66 & $\mathrm{a}$ & B 1,09 & $\mathrm{~B}$ & 1,37 \\
\hline 5,0 & A 2,09 & $\mathrm{a}$ & B 1,36 & $\mathrm{ab}$ & 1,72 \\
\hline 10,0 & A 1,83 & $\mathrm{a}$ & A 1,59 & $\mathrm{ab}$ & 1,71 \\
\hline 15,0 & A 1,95 & $\mathrm{a}$ & A 1,87 & $\mathrm{a}$ & 1,91 \\
\hline 20,0 & A 1,54 & $\mathrm{a}$ & A 1,41 & $\mathrm{ab}$ & 1,47 \\
\hline 25,0 & A 1,66 & $\mathrm{a}$ & A 1,47 & $\mathrm{ab}$ & 1,56 \\
\hline Média & 1,79 & & 1,47 & & \\
\hline $\mathrm{CV}$ & & & $24,05 \%$ & & \\
\hline $\mathrm{cm}$ & \multicolumn{5}{|c|}{ Ponto de murcha permanente } \\
\hline 2,5 & A 5,49 & $a b$ & B 2,87 & $\mathrm{c}$ & 4,18 \\
\hline 5,0 & A 5,98 & $\mathrm{a}$ & B 3,52 & $\mathrm{bc}$ & 4,75 \\
\hline 10,0 & A 5,64 & $\mathrm{ab}$ & A 4,68 & $\mathrm{a}$ & 5,16 \\
\hline 15,0 & A 5,25 & $a b c$ & B 3,68 & $\mathrm{bc}$ & 4,46 \\
\hline 20,0 & A 4,94 & $\mathrm{c}$ & B 3,94 & $\mathrm{ab}$ & 4,44 \\
\hline 25,0 & A 4,33 & $\mathrm{c}$ & A 3,54 & $\mathrm{bc}$ & 3,93 \\
\hline Média & 5,27 & & 3,71 & & \\
\hline $\mathrm{CV}$ & $16,20 \%$ & & & & \\
\hline
\end{tabular}

${ }^{1}$ Plantio direto

${ }^{2}$ Plantio direto escarificado

Médias antecedidas pela mesma letra maiúscula na horizontal e seguidas pela mesma letra minúscula na vertical não diferem pelo teste de Duncan a 5\% de probabilidade de erro. 
superior ao PDE, destacando-se o aumento da diferença com a redução da umidade do solo. Isto demonstra a eficiência da escarificação na redução da resistência mecânica do solo, concordando com INOUE et al. (2002) e contradizendo RALISCH et al. (2001), que verificaram o efeito da redução na RP no espaço de tempo durante um ciclo de cultura anual, concluindo que o efeito da escarificação na RP não perdura por mais do que um cultivo.

Na umidade do solo na capacidade de campo (Tabela 2), a RP no PD foi superior ao PDE, não apresentando diferença significativa entre as profundidades. No entanto, o PDE apresentou uma variação significativa, sendo a menor RP na profundidade $2,5 \mathrm{~cm}$, e a maior na profundidade de $15 \mathrm{~cm}$, enquanto nas demais não diferiram. Entre os sistemas de manejo em profundidade, observou-se diferença significativa somente nas profundidades de 2,5 e 5,0cm, tendo o PD apresentado a RP superior ao PDE, ao passo que nas demais não apresentaram diferença significativa.

A condição de umidade do solo no ponto de murcha permanente (Tabela 2) foi a que apresentou a maior diferença de RP entre os sistemas de manejo de solo. O PD apresentou uma RP maior que a do PDE, demonstrando a influência da escarificação em diminuir a limitação mecânica do crescimento do sistema radicular das plantas em condições de deficiência hídrica. Em profundidade no PD, a maior RP foi de 2,5 a $15,0 \mathrm{~cm}$, enquanto nas profundidades de 20 e $25 \mathrm{~cm}$, constatou-se a menor RP. No PDE, a RP das profundidades de 10 e $20 \mathrm{~cm}$ foi superior as demais, e na profundidade de $2,5 \mathrm{~cm}$ constatou-sea menor $\mathrm{RP}$ entre às profundidades.

Comparando a RP entre manejos em profundidades, o PDE foi significativamente inferior nas profundidades de 2,$5 ; 5 ; 15$ e $20 \mathrm{~cm}$, ao passo que, na profundidade de 10 e $25 \mathrm{~cm}$, os manejos de solo não diferiram estatisticamente.

Os componentes de rendimento da soja e a altura das plantas avaliadas não apresentaram diferença significativa, tanto para os sistemas de manejo do solo como para os tipos de sulcadores de semeadora-adubadora (Tabela 3), indicando um desenvolvimento muito similar das plantas da soja em condições de solo distintas.

O rendimento de grãos da cultura da soja (Tabela 3) não apresentou diferença significativa entre os manejos de solo adotado. Este mesmo resultado foi observado por BOLLER (1990) e CENTURION \& DEMATTÊ (1992), que pode ser atribuído à precipitação pluvial de $999,8 \mathrm{~mm}$ durante o ciclo da cultura (132 dias) que contribui para manter a umidade do solo nas condições ideais, minimizando o efeito da compactação.

Em contrapartida aos resultados obtidos neste trabalho, vários estudos demonstram que a escarificação promove um incremento no rendimento das culturas devido à redução da resistência à penetração das raízes e densidade do solo e um aprofundamento do sistema radicular (PAULETTI, 2002).

Os sulcadores apresentaram influência no rendimento de grãos da cultura da soja, no sistema $\mathrm{PD}$, tendo o sulcador $\mathrm{G}$ obtido um maior rendimento de grãos com $3126 \mathrm{~kg} \mathrm{ha}^{-1}$ contra $2984 \mathrm{~kg} \mathrm{ha}^{-1}$ do DD. Isso pode ser atribuído ao fato do mecanismo tipo $\mathrm{G}$ ter uma maior capacidade de penetração, podendo romper as camadas compactadas que são comumente encontradas em solos argilosos, minimizando os efeitos da densidade do solo e melhorando o leito de semeadura, favorecendo a cultura, enquanto que o DD somente abre o solo para a deposição do adubo e da semente (SILVA et al., 2001). No PDE, os mecanismos sulcadores não apresentaram influência no rendimento

Tabela 3 - Estatura de plantas, massa de 1000 grãos, número de vagens por planta, número de grãos por vagem e rendimento de grãos de soja em função do manejo do solo e de mecanismos sulcadores de semeadura

\begin{tabular}{|c|c|c|c|c|c|c|}
\hline $\begin{array}{l}\text { Manejo } \\
\text { do solo }\end{array}$ & Mecanismo sulcador & $\begin{array}{l}\text { Estatura plantas } \\
\mathrm{cm}\end{array}$ & $\begin{array}{c}\text { Massa } 1000 \text { grãos } \\
\text { g }\end{array}$ & $\mathrm{N}^{\mathrm{o}}$ vagens por planta & $\mathrm{N}^{\circ}$ grãos por vagem & $\begin{array}{l}\text { Rendimento de grãos } \\
\mathrm{kg} \mathrm{ha}^{-1}\end{array}$ \\
\hline \multirow{2}{*}{$\mathrm{PD}^{1}$} & $\mathrm{DD}^{3}$ & 92,8 & 136,9 & 31 & 2,00 & 2984 b \\
\hline & $\mathrm{G}^{4}$ & 94,0 & 141,0 & 28 & 2,07 & $3126 \mathrm{a}$ \\
\hline \multirow{2}{*}{$\mathrm{PDE}^{2}$} & DD & 91,8 & 138,4 & 32 & 2,10 & 2991 \\
\hline & $\mathrm{G}$ & 92,8 & 138,9 & 31 & 2,09 & 2980 \\
\hline
\end{tabular}

${ }^{1}$ Plantio direto

${ }^{2}$ Plantio direto escarificado

${ }^{3}$ Sulcador da semeadora adubadora do tipo duplo disco

${ }^{4}$ Sulcador da semeadora adubadora do tipo guilhotina

Médias seguidas por letras diferentes na vertical diferem pelo teste de Duncan a 5\% de probabilidade de erro.

Ciência Rural, v.35, n.4, jul-ago, 2005. 
da soja. O efeito do tipo sulcador em PD também foi observado por KLEIN \& BOLLER (1995) que obtiveram um rendimento na cultura do milho de $30 \%$ superior com sulcador $\mathrm{G}$ se comparado com o sulcador DD.

\section{CONCLUSÕES}

A escarificação esporádica em solos sob plantio direto proporciona condições físico-hídricamecânicas do solo mais favoráveis ao desenvolvimento das plantas, especificamente pela redução na resistência mecânica à penetração e pela não redução do teor de matéria orgânica, sendo que as possíveis limitações estudadas não afetaram o desenvolvimento e rendimento de grãos da cultura da soja, em função da precipitação pluvial favorável durante todo o ciclo da cultura. O mecanismo sulcador do tipo guilhotina proporcionou aumento no rendimento da soja sob plantio direto.

\section{REFERÊNCIAS}

BAYER, C.; MIELNICZUK, J. Características químicas do solo afetadas por métodos de preparo e sistemas de culturas. Revista Brasileira de Ciência do Solo, v.21, n.1, p.105112, 1997.

BOLLER, W. Desenvolvimento de complementos para semeadoras em solo sob preparo reduzido. 1990. 146f. Dissertação (Mestrado em Engenharia Agrícola) - UFSM.

BRAIDA, J.A. et al. Carbono orgânico e suas implicações na umidade crítica para a compactação do solo. In: CONGRESSO BRASILEIRO DE CIÊNCIA DO SOLO, 29., 2003, Ribeirão Preto. Resumo expandido... Ribeirão Preto : SBCS, 2003. CD-ROM.

BUSSCHER, W.J. Adjustment of flat-tipped penetrometer resistance dta to a common water content. Transactions of ASAE, v.33, n.2, p.519-524, 1990.

CAMARGO, D.A.; ALLEONI, L.R.F. Compactação do solo e desenvolvimento de plantas. Piracicaba : USP/ESALQ, 1997. 132p.

CARVALHO, E.J. et al. Comportamento físico-hídrico de um Podzólico Vermelho-amarelo câmbico fase terraço sob diferentes sistemas de manejo. Pesquisa Agropecuária Brasileira, v.34, n.2, p.257-265, 1999.

CARTER, M.R. Relative measures of soil bulk density to characterize compaction in tillage studies of fine loamy sands. Canadian Journal of Soil Science, v.70, p.425-433, 1990.

CENTURION, J.F.; DEMATTÊ, J.L.I. Sistema de preparo de solos de cerrado: Efeitos nas propriedades físicas e na cultura do milho. Pesquisa Agropecuária Brasileira, v.27, n.2, p.315-324, 1992.

DIAS JUNIOR, M.S.; MIRANDA, E.E.V. de. Comportamento da curva de compactação de cinco solos da região de Lavras (MG). Ciência Agrotécnica, v.24, p.337-346, 2000.
EMPRESA BRASILEIRA DE PESQUISA AGROPECUÁRIA. Centro Nacional de Pesquisa de Solos. Manual de métodos de Análise de Solo. 2.ed. Rio de Janeiro 1997. 212p.

FERRERAS, L.A. et al. Parâmetros físicos del suelo em condiciones no perturbadas y bajo laboreo. Pesquisa Agropecuária Brasileira, v.36, n.1, p.161-170, 2001.

FREITAS, P.L., de. Aspectos físicos e biológicos do solo. In: LANDERS, J.N. (Ed). Experiências de plantio direto no Cerrado. Goiânia : APDC, 1994. p.199-213. 261p.

GOEDERT, W. J.et al. Estado de compactação do solo em áreas cultivadas no sistema de PD. Pesquisa Agropecuária Brasileira, v.37, n.2, p.223-227, 2002.

INOUE, T.T. et al. Influência da escarificação em propriedades físicas de um Latossolo vermelho distroférrico após 13 anos de PD. In: REUNIÃO BRASILEIRA DE MANEJO E CONSERVAÇÃO DO SOLO E DA ÁGUA, 14., 2002, Cuiabá. Resumos Expandidos... Cuiabá : SBCS, 2002. CD-ROM.

KAY, V.A. Potential indicators of the quality of soil structure for plant growth. In: WORKSHOP INTERNACIONAL EM AVANÇOS EM CIÊNCIA DO SOLO: A FÍSICA DO SOLO NA PRODUÇÃO AGRÍCOLA E QUALIDADE AMBIENTAL. Disponível na internet: http://www.esalq.usp.br/lsn/ work_pal.htm. Online. Acesso: 13 de outubro de 2000.

KLEIN, V.A. Densidade relativa - um indicador de qualidade física do solo. In: REUNIÃO BRASILEIRA DE MANEJO E CONSERVAÇÃO DO SOLO E DA ÁGUA, 14., 2002. Cuiabá. Resumos Expandidos... Cuiabá : SBCS, 2002. CD-ROM.

KLEIN, V.A.; BOLLER, W. Avaliação de diferentes métodos de manejos de solo e métodos de semeadura em áreas sob PD. Ciência Rural, Santa Maria, v. 25, n.3, p.395-398, 1995.

KLEIN, V. A.; LIBARDI, P. L. Faixa de umidade menos limitante ao crescimento vegetal e a sua relação com a densidade do solo ao longo do perfil de um Latossolo Roxo. Ciência Rural, v.30, n.6, p.959-964, 2000.

KOCHHANN, R.A.; DENARDIN, J.E. Implantação e manejo do sistema plantio direto. Passo Fundo : EMBRAPA-CNPT, 2000. 36p.

MACHADO, R.L.T. et al. Estudos de parâmetros físicos em solo de várzea, entes e após escarificação. Revista Brasileira de Agrociência, v.2, n.3, p.175-178, 1996.

NOGUEIRA, J.B. Mecânica dos solos:ensaios de laboratório. São Carlos : USP, EESC, 1998. 248p.

PAULETTI, V. Efeitos de métodos de preparo do solo sobre a produção de grãos das principais culturas e características químicas do solo, na região do $\mathrm{ABC}$. Disponível em: http://www.fundacaoabc.com.br/artigos/ fertilidade/fertil2_98.htm. Online. acesso em: 01 jul. 2002.

PIERCE, F.J. et al. Immediate and residual effects of zonetillage in rotation with no-tillage on soil physical properties and corn performance. Soil \& Tillage Research, v.30, p.149-165. 1992.

RALISCH, R. et al. Avaliação de um solo argiloso sob PD de uma escarificação na evolução da resistência do solo a penetração. In: CONGRESSO BRASILEIRO DE 
ENGENHARIA AGRÍCOLA, 30., 2001, Foz do Iguaçu. Resumo expandido... Foz do Iguaçu : CONBEA, 2001. CD-ROM.

SECCO, D.; REINERT, D.J. Efeitos imediato e residual de escarificadores em Latossolo Vermelhoescuro sob PD. Engenharia Agrícola, v.16, n.3, p.52$61,1997$.

SECCO, D. et al. Efeito de sistemas de manejo nas propriedades físicas de um Latossolo Vermelho-escuro. In: REUNIÃO SUL-BRASILEIRA DE CIÊNCIA DO SOLO, 1., 1996. Lages. Resumo expandido... Lages : SBCS, 1996. p.127-128.
SILVA, A.R.B. et al. Avaliação de uma semeadora-adubadora de plantio direto em função de diferentes mecanismos sulcadores e velocidades de deslocamento. In: CONGRESSO BRASILEIRO DE ENGENHARIA AGRÍCOLA, 30., 2001, Foz do Iguaçu. Resumo expandido... Foz do Iguaçu : CONBEA, 2001. CD-ROM.

TEDESCO, M.J. et al. Análise de solo, plantas e outros minerais. Porto Alegre : Departamento de solos - Faculdade de Agronomia, UFRGS, 1995. 174p.

TORMENA, C.A.; ROLOFF, O.F. Dinâmica da resistência a penetração de um solo sob PD. Revista Brasileira de Ciência do Solo, v.20, n.2, p.333-339, 1996. 